\title{
Bond Strength of Orthodontic Bracket Cement Using a Bleaching Light for Curing
}

\author{
Yoav Shapinko ${ }^{*}$, Iro Eleftheriadi ${ }^{*}$, Nir Shpack1, Moshe Davidovitch ${ }^{1}$, \\ Elias Bitsanis' ${ }^{2}$, Shlomo Matalon ${ }^{3}$, Tamar Brosh ${ }^{1,4}$
}

\author{
${ }^{1}$ Department of Orthodontics, Maurice and Gabriela Goldschleger School of Dental Medicine, Tel Aviv University, \\ Tel Aviv, Israel \\ ${ }^{2}$ Department of Orthodontics, School of Dentistry, National and Kapodistrian University of Athens, Athens, Greece \\ ${ }^{3}$ Department of Oral Rehabilitation, Maurice and Gabriela Goldschleger School of Dental Medicine, Tel Aviv University, \\ Tel Aviv, Israel \\ ${ }^{4}$ Department of Oral Biology, Maurice and Gabriela Goldschleger School of Dental Medicine, Tel Aviv University, \\ Tel Aviv, Israel \\ Email:*elefiro@gmail.com
}

How to cite this paper: Shapinko, Y., Eleftheriadi, I., Shpack, N., Davidovitch, M., Bitsanis, E., Matalon, S. and Brosh, T. (2018) Bond Strength of Orthodontic Bracket Cement Using a Bleaching Light for Curing. Open Journal of Stomatology, $\mathbf{8}$, 81-89.

https://doi.org/10.4236/ojst.2018.83007

Received: January 29, 2018

Accepted: March 17, 2018

Published: March 20, 2018

Copyright $\odot 2018$ by authors and Scientific Research Publishing Inc. This work is licensed under the Creative Commons Attribution International License (CC BY 4.0).

http://creativecommons.org/licenses/by/4.0/

(c) (i) Open Access

\begin{abstract}
Aim: To investigate the bond strengths achieved by using a Bleaching Curing Light (BCL) to polymerize orthodontic bonding cement. Material and Methods: 160 anterior bovine teeth were used to form 20 average sized human dental arches, and distributed into 2 groups according to which light curing method used: Group 1: BCL for 40 seconds, or Group 2: LED for 10 seconds. After storage in a controlled environment, Shear Bond Strength (SBS) and Adhesive Remnant Index (ARI) were determined. Results: Group 1 showed significantly lower SBS in the most posterior (first molar) position of the dental arch, (Group 1: $0.7 \pm 1.0 \mathrm{MPa}$, Group 2: $2.9 \pm 1.7 \mathrm{MPa}, \mathrm{p}<0.01$ ), but significantly higher SBS in the most anterior position (Group 1: $5.1 \pm 1.8 \mathrm{MPa}$, Group 2: $3.8 \pm 1.2 \mathrm{MPa}, \mathrm{p}<0.02)$. A high correlation was found between the position of the bracket and debonding values $(p<0.02)$. Bonding failures in the most posterior arch positions occurred more frequently within Group 1, and lower ARI than Group 2 over corresponding arch locations. Conclusion: Simultaneous full-arch curing of orthodontic bracket cement using a BCL is clinically acceptable in all but the most posterior locations along the dental arch.
\end{abstract}

\section{Keywords}

Bleaching, Light-Cure, Orthodontic Bonding, Shear Bond Strength

*Yoav Shapinko and Iro Eleftheriadi are joint first authors and contributed equally to this work. 


\section{Introduction}

In the clinical practice of orthodontics, bonding of fixed appliances is one of the most time-consuming tasks. Hence, ergonomic measures such as: combined agents, pre-coated brackets, reduced curing time, and indirect bonding procedures have been proposed [1]. In addition, the use of an enlarged light-exiting tip has been reported to develop shear bond strength equal or lower to a conventional tip [2].

Whereas, in vivo investigations of bond strength present difficulties in investigating independent variables, in vitro human and bovine models pervade the literature, although varied in experimental design [3] [4] [5]. Concomitantly, the use of light curing methods [6] [7] varied adhesive materials or bonding methods [8] [9], and debonding procedures have also been reported [10].

The advent of light-catalyzed vital dental bleaching [11], has provided a cross-over tool which may improve the ergonomics of orthodontic appliance bonding by facilitating the simultaneous curing over an entire arch.

The purpose of this study was to investigate the efficiency of a Bleaching Curing Light (BCL) in simulated one-arch orthodontic bracket bonding. The null hypothesis being that this will produce similar results compared to current methods.

\section{Materials and Methods}

\subsection{Study Design}

160 intact anterior bovine teeth were harvested from beef carcasses, and preserved in Thymol [12]. The inclusion criteria were that the teeth were permanent and that the buccal surfaces were caries-free, so that primary bovine teeth or teeth with decayed buccal surfaces were excluded. These were arranged into 20 arches with their roots in wax bases (Dentaurum, Ispringen, Germany) to correspond to the largest average human dental arch [13]. Samples were distributed so that half of the 8 teeth originating from each source were included in the experimental and half in the control groups. As shown in Figure 1 and in Figure 2, sample position denoted using the ISO 3950:2016 (FDI) system of notation [14] and each arch was oriented within a dental manikin (Columbia Dentoform, New York, USA).

These were divided into two equal groups: Group 1, 40 second static exposure to LED Bleaching Curing Light (BCL) (Figure 2), Group 2, 10 second separate exposure of each bracket to a LED Regular Tip curing light (RT) (both from Foshan Coxo Medical Instrument CO, LTD, Guangdong, China). The exposure time in both groups was based on manufacturer's instructions of use.

Prior to light curing, all teeth were cleaned with Zircate Prophy Paste (Dentsply, Milford, USA) for 15 seconds, and debrided by washing for 5 seconds with water spray, then dried with oil-free compressed air. The labial surfaces where adhesive bonding was to be applied were then prepared using 37\% 


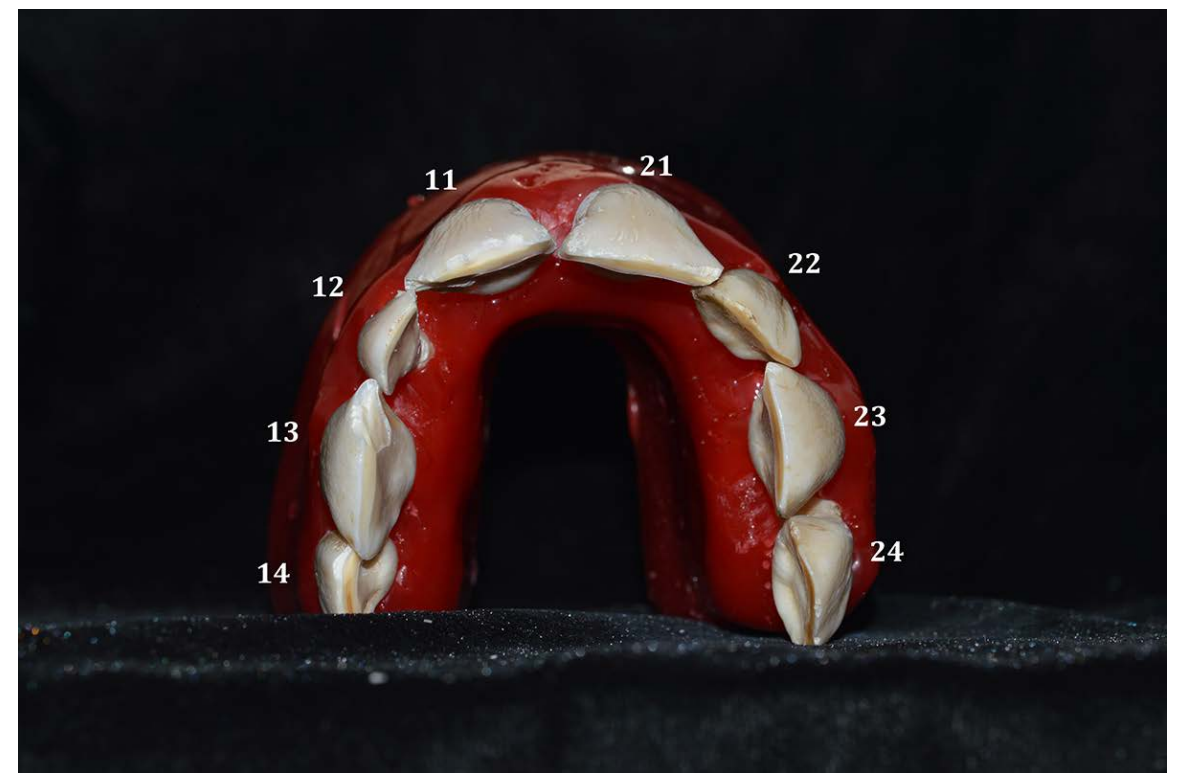

Figure 1. Teeth arrangement in the constructed arches analogous to the FDI system. (Bonding Position 1: \#11,21, Bonding Position 2: \#12,22, Bonding position 3: \#13,23, Bonding Position 4: \#14,24\}.

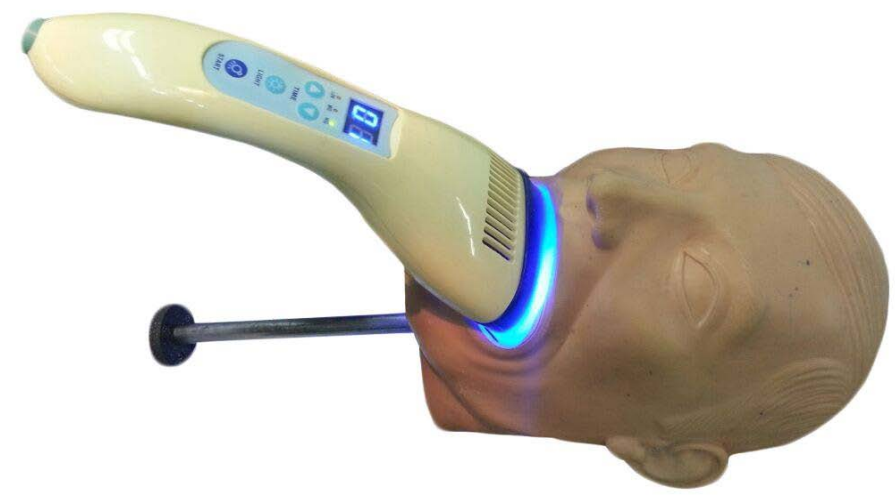

Figure 2. Intraoral adjustment of the BCL.

ortho-phosphoric acid for 30 seconds (Vista TM, Racine, Wisconsin, USA), according to Saleh [15], and debrided as above.

The prepared enamel surfaces were bonded according to manufacturer instructions using XT Primer, and Transbond XT Composite (3M, Unitek, Monrovia California USA) [16]. The latter being applied to the mesh pads of premolar brackets (Hangzhou ORJ, China) which were oriented so that the most posterior bracket on each side approximated the position of a first molar tube, based on mean tooth widths [13]. The 3 more anterior brackets were positioned so that full contact was achieved between the bracket base the prepared enamel surface. The bracket bonding material was polymerized as described above with either the BCL or conventional curing light, after which all casts were removed from the mannequins and stored at $85 \%$ humidity and $37^{\circ} \mathrm{C}$ for 24 hours.

Samples were removed from their wax base and each positioned in a holding 
device positioning its buccal surface parallel to the direction of a loading force applied via a 5-strand braided $0.0195 "$ stainless steel wire (Ortho Organizers, Carlsbad, USA) around the wings of each bracket held by an Instron, Model 4502 (Buckinghamshire, England) formatted with a $10 \mathrm{kN}$ load-cell, applied at $10 \mathrm{~mm} / \mathrm{min}$ cross-head speed. SBS was calculated by dividing the debonding force (measured force causing debonding), by the area of the bracket base.

Following debonding, all bracket bases were inspected under 10× stereoscopic magnification (Wild, Heerbrugg, Switzerland), and a Adhesive Remnant Index (ARI) determination was made according to Kapur et al. [17]. This was graded as:

0 : no adhesive present on the bracket base.

1: $0 \%<$ ARI $<50 \%$.

2: $50 \%<\mathrm{ARI}<75 \%$.

3: ARI $>75 \%$.

\subsection{Statistical Analysis}

ANOVA with repeated measures and paired t-test were carried out to compare differences between the two groups according to position along the arch. Kruskal Wallis test was used to determine any significant differences in scaled ARI values in the various bonding positions within each group. Wilcoxon Signed Ranks Test was used to determine the differences of ARI in the various bonding positions between the two groups. Pearson correlation was applied to verify correlations between SBS and ARI. Statistical significance for all tests was considered as $\mathrm{p}<0.05$.

This research project was approved by the Ethics Committee of the institution where it was held.

\section{Results}

The mean SBS values of the two groups according to position along the arch are presented in Table 1. Bonding failures (debonding force $=0 \mathrm{~N}$ ), were found in teeth 14 and 24 in Group 1. The mean SBS of this position in Group $1(0.7 \pm 1.0$ $\mathrm{MPa})$ was significantly lower $(\mathrm{p}<0.001)$ than those of other positions along the simulated dental arch.

Data related to position in the arch and type of curing on the debonding force is shown in Figure 3. A high correlation was found between the position of the bracket on the dental arch and the debonding values $(\mathrm{p}<0.002)$. SBS in teeth 11.21 of Group 1 were significantly higher than in group $2(\mathrm{p}<0.02)$, whereas in teeth 14.24 Group 2 showed 3.8-fold higher SBS ( $\mathrm{p}<0.001$ ). In positions 2 and 3 the differences between the two groups were not significant $(\mathrm{p}>0.05)$ (Figure 3).

The ARI within group 1 was found to vary statistically according to position $(\mathrm{p}=0.017)$ (Table 2). The Wilcoxon Signed Ranks Test showed significant differences in positions $3(\mathrm{p}=0.032)$ and $4(\mathrm{p}=0.030)$ between groups 1 and 2 


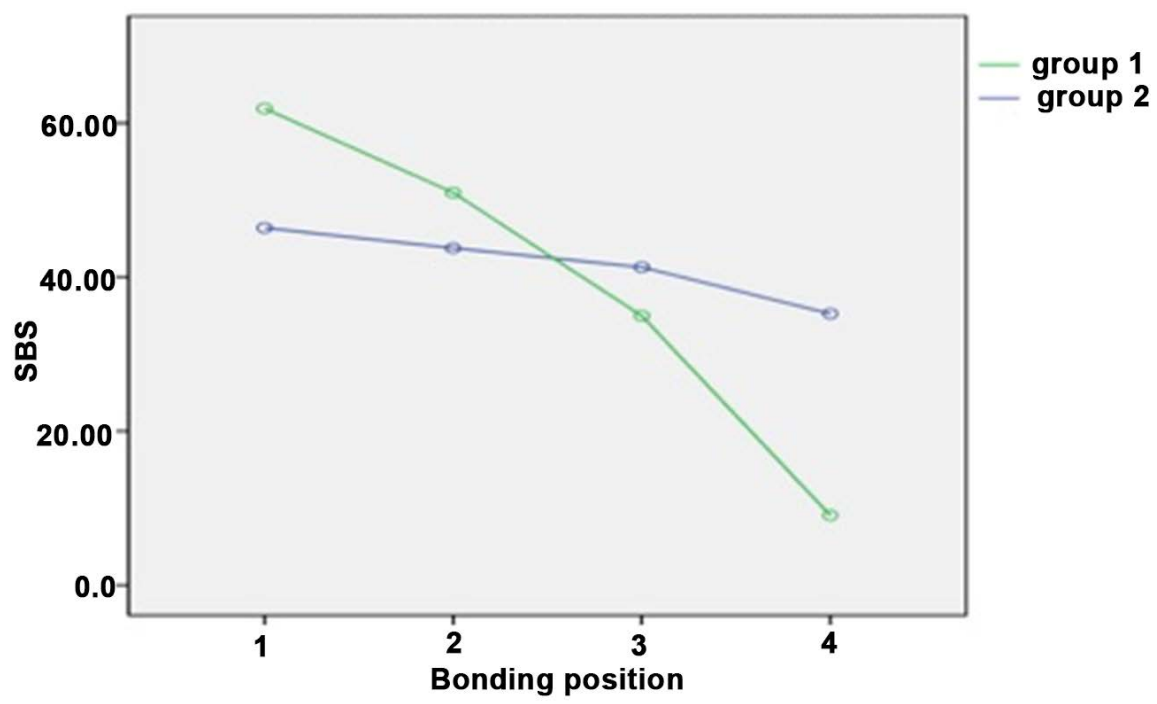

Figure 3. SBS values in group 1 (green) and group 2 (blue).

Table 1. Mean SBS and debonding force values and standard deviations for the two groups according to bonding position along the arch.

\begin{tabular}{cccc}
\hline & Bonding Position & SBS (MPa) & Mean Debonding Force (N) \\
\hline Group 1 $(\mathrm{n}=20)$ & 1 & $5.1 \pm 1.8$ & $61.9 \pm 22.6$ \\
& 2 & $4.2 \pm 1.9$ & $51.0 \pm 23.3$ \\
& 3 & $2.9 \pm 1.7$ & $34.9 \pm 20.5$ \\
Group 2 $(\mathrm{n}=20)$ & 4 & $0.7 \pm 1.0$ & $9.1 \pm 12.5$ \\
& 1 & $3.8 \pm 1.2$ & $46.4 \pm 14.2$ \\
& 2 & $3.6 \pm 1.7$ & $43.8 \pm 20.4$ \\
& 3 & $3.4 \pm 1.4$ & $41.3 \pm 16.6$ \\
& 4 & $2.9 \pm 1.7$ & $35.2 \pm 20.6$ \\
\hline
\end{tabular}

Table 2. Kruskal-Wallis test examining differences in scaled ARI values between different bonding positions within each group.

\begin{tabular}{ccc}
\hline & Group 1 & Group 2 \\
\hline Chi-square & 10.224 & 1.608 \\
$\mathrm{df}$ & 3 & 3 \\
$\mathrm{p}$-value & 0.017 & 0.658 \\
\hline
\end{tabular}

(Table 3). However, no significant correlations were found between the SBS and ARI in any group.

\section{Discussion}

The present study found that the use of the BCL resulted in much lower SBS values in the most posterior position, rejecting the null hypothesis. However, 
Table 3. Wilcoxon Signed Ranks Test examining the differences of ARI in the various bonding positions between the two groups.

\begin{tabular}{ccccc}
\hline & Position 4 & Position 3 & Position 2 & Position 1 \\
\hline $\mathrm{Z}$ & -2.174 & -2.144 & -0.464 & -0.061 \\
Asymp. Sig. (2-tailed) & 0.030 & 0.032 & 0.642 & 0.951 \\
\hline
\end{tabular}

samples located anteriorly showed similar inter-group SBS values, implying that this method of activation is appropriate but location sensitive.

The multiple failures of bonding in the most posterior position in Group 1, together with the much smaller mean value of debonding force, suggests clinically that bonding to the first molars using the BCL will be least successful. Since the bonding protocol was the same for all the other steps of the bonding procedure, this finding must be due to insufficient (light) curing of the bracket adhesive material in the posterior region of the arch.

Although, effects of the BCL during dental bleaching has been previously reported [11] [18], there are no studies reporting comparisons between the anterior and posterior aspects of the dental arch. Differences in SBS related to position along the arch might be explained by the shape and intended use of the BLC making distribution of light less efficient posteriorly.

In Group 1, SBS ranged from 0.2 to $11.5 \mathrm{MPa}$ (excluding bond failures), which are lower than those previously reported [9] [19]. This is likely due to differences in study designs, materials tested, methods used for the measurements, or specimens differences. Furthermore, methodological variations such as consistency of lever-arm point of force application, or thickness of adhesive layer are innate to such investigations. As a result, there is an additional torque acting that is ignored during debonding tests [3].

Clinically acceptable bond strengths range between 5.9 and 7.8 MPa [20]. Deceasing SBS posteriorly in Group 1 (Figure 2), but significantly higher debonding strength in teeth 11, 21 compared to Group 2 may be due to the longer curing time (40 seconds versus 10 seconds, respectively). It has been previously reported that increasing exposure $(5,10$ or 15 seconds) with the same LED did not cause significant differences in SBS, but average values were found to be higher with longer curing time [21]. However, it was not the purpose of this study to base clinical conclusions on an in vitro shear bond strength experiment, due to the well known methodological problems associated with the design of such tests [3] [22].

In positions 2 and 3 the differences in SBS between the two groups were not statistically significant. This suggests that successful bonding can be achieved when using the BCL also in the premolar area. However, bond failures found in Group 1 decreased the SBS mean at position 4 (3.34 MPa). Excluding these, the SBS values found in the anterior and premolar areas may be considered within the required range.

The use of bovine teeth as an appropriate in vitro dental model has been pre- 
viously reported [23] [24] [25]. It has been shown that both shear and tensile strengths are not significantly different between human and bovine dentin [26] [27], or enamel [27]. In addition, reported dental bond strengths in human and bovine studies conclude that the latter can substitute for human teeth in in vitro studies establishing the initial performance of new products [9] [28] [29].

The latency period of 24 hours after bonding has been reported to increase the setting time of light-cured adhesives [30] [31]. This has been associated with the increase in shear strength reported when allowing setting for 24 hours [32] to 7 days [33]. However, these do not correspond to clinical reality, where brackets are loaded immediately after bonding, therefore, for purposes of comparison, a 24-hour latency period was adopted for the present study [32] [34].

No significant correlation was found between ARI and bond strength within each group, in agreement with Linn et al [35]. However, significant differences were detected between the two groups in positions $3(\mathrm{p}=0.032)$ and $4(\mathrm{p}=$ 0.030 ), in which the study group showed lower ARI values, and it was found that adhesive bond fracture occurred between the adhesive and the bracket base more frequently in the study group. This implies a tendency for greater amounts of residual composite in the posterior areas at bracket removal when the BCL was used. However, this requires further investigation since here the bracket/adhesive interface was determined only by visual inspection.

\section{Conclusions}

1) Light curing with the BCL leads to similar polymerization of orthodontic adhesive in the anterior and premolar regions.

2) The SBS values suggest that curing with the BCL is an appropriate but location sensitive activation method, thus it would not be effective for one-arch orthodontic bonding.

\section{References}

[1] Silverman, E., Cohen, M., Gianelly, A. and Diez, V. (1972) A Universal Direct Bonding System for Metal and Plastic Brackets. American Journal of Orthodontics, 62, 236-244. https://doi.org/10.1016/S0002-9416(72)90264-3

[2] Oesterle, L.J., Newman, S.M. and Shellhart, W.C. (2002) Comparative Bond Strength of Brackets Cured Using a Pulsed Xenon Curing Light with Two Different Light-Guide Sizes. American Journal of Orthodontics and Dentofacial Orthopedics, 122, 242-250. https://doi.org/10.1067/mod.2002.126728

[3] Fox, N.A., McCabe, J.F. and Buckley, J.G. (1994) A Critique of Bond Strength Testing in Orthodontics. British Journal of Orthodontics, 21, 33-43. https://doi.org/10.1179/bjo.21.1.33

[4] Hajrassie, M.K.A. and Khier, S.E. (2007) In-Vivo and In-Vitro Comparison of Bond Strengths of Orthodontic Brackets Bonded to Enamel and Debonded at Various Times. American Journal of Orthodontics and Dentofacial Orthopedics, 131, 384-390. https://doi.org/10.1016/j.ajodo.2005.06.025

[5] Bakhadher, W., Halawany, H., Talic, N., Abraham, N. and Jacob, V. (2015) Factors Affecting the Shear Bond Strength of Orthodontic Brackets-A Review of in Vitro 
Studies. Acta Medica, 58, 43-48.

[6] Carvalho, P.E.G., Muzulon dos Santos, V., Isber, H. and Cotrim-Ferreirar, F.A. (2013) Halogen Light versus LED for Bracket Boning: Shear Bond Strength. Dental Press Journal of Orthodontics, 18, 31.e1-e6. https://doi.org/10.1590/S2176-94512013000100007

[7] Magno, A.F.F., Martins, R.P., Vaz, L.G. and Martins, L.P. (2010) In Vitro Lingual Bracket Evaluation of Indirect Bonding with Plasma Arc, LED and Halogen Light. Orthodontics \& Craniofacial Research, 13, 48-55. https://doi.org/10.1111/j.1601-6343.2009.01474.x

[8] Fernandes, H.O., Santos, I.B. and Firoozmand, L.M. (2015) Shear Bond Strength of an Orthodontic Self-Etching Adhesive after Intracoronary Bleaching. Orthodontics \& Craniofacial Research, 18, 117-124. https://doi.org/10.1111/ocr.12067

[9] Dominguez, A., Garcia, J.A., Costela, A. and Gomez, C. (2011) Influence of the Light Source and Bleaching Gel on the Efficacy of the Tooth Whitening Process. Photomedicine and Laser Surgery, 29, 53-59. https://doi.org/10.1089/pho.2009.2751

[10] Ajaj, A.R., Chiappelli, F., Phi, L., Giroux, A., Maida, C., Garrett, N. and Polinovsky, O. (2012) Evidence-Based Assessment of the Efficacy and Effectiveness of Light/Laser Activation in In-Office Dental Bleaching. Dental Hypotheses, 3, 55-66. https://doi.org/10.4103/2155-8213.100388

[11] Romano, F.L., Correr, A.B., Sobrinho, L.C., Magnani, M.B.B.A. and Siquieira, V.C.V. (2009) Shear Bond Strength of Metallic Brackets Bonded with a New Orthodontic Composite. Brazilian Journal of Oral Sciences, 8, 76-80.

[12] Maranhao, K.M., Klatau, E.B., Pereira, P.M.M., Guimaraes, R.B. and Pantoja, V.G. (2009) The Effect of Storage Solutions on Enamel of Bovine Teeth. Salusvita, 28, 129-134.

[13] Howe, R.P., McNamara, J.A. and O'Connor, K. (1983) An Examination of Dental Crowding and Its Relationship to Tooth Size and Arch Dimension. American Journal of Orthodontics, 83, 363-373. https://doi.org/10.1016/0002-9416(83)90320-2

[14] Elderton, R.J. (1989) Keeping Up-to-Date with Tooth Notation. British Dental Journal, 166, 55-56. https://doi.org/10.1038/sj.bdj.4806709

[15] Saleh, F.K. (2005) Variations in Human and Bovine Surface Enamel Acid Etching Patterns and Resin Penetration: A Scanning Electronic Microscopy In-Vitro Study. Lebanese Science Journal, 6, 1-13.

[16] Bishara, S.E., Soliman, M., Laffoon, J. and Warren, J.J. (2005) Effect of Changing a Test Parameter on the Shear Bond Strength of Orthodontic Brackets. The Angle Orthodontist, 75, 832-835.

[17] Kapur, R., Sinha, P.K. and Nanda, R.S. (1999) Comparison of Load Transmission and Bracket Deformation between Titanium and Stainless Steel Brackets. American Journal of Orthodontics and Dentofacial Orthopedics, 116, 275-278. https://doi.org/10.1016/S0889-5406(99)70238-6

[18] Joiner, A. (2006) The Bleaching of Teeth: A Review of the Literature. Journal of Dentistry, 34, 412-419. https://doi.org/10.1016/j.jdent.2006.02.002

[19] Di Nicolo, R., Araujo, M.A.M., Alves, L.A.C., Souza, R.O.A. and Rocha, D.M. (2010) Shear Bond Strength of Orthodontic Brackets Bonded using Halogen Light and Light-Emitting Diode at Different Debond Times. Brazilian Oral Research, 24, 64-69. https://doi.org/10.1590/S1806-83242010000100011

[20] Reynolds, I.R. (1975) A Review of Direct Orthodontic Bonding. British Journal of Orthodontics, 2, 171-178. https://doi.org/10.1080/0301228X.1975.11743666 
[21] Dall'Igna, C.M., Marchioro, E.M., Spohr, A.M. and Mota, E.G. (2011) Effect of Curing Time on the Bond Strength of a Bracket-Bonding System Cured with a Light-Emitting Diode or Plasma Arc Light. European Journal of Orthodontics, 33, 55-59. https://doi.org/10.1093/ejo/cjq027

[22] Eliades, T. and Brantley, W.A. (2000) The Inappropriateness of Conventional Orthodontic Bond Strength Assessment Protocols. European Journal of Orthodontics, 22, 13-23. https://doi.org/10.1093/ejo/22.1.13

[23] Yassen, G.H., Platt, J.A. and Hara, A.T. (2011) Bovine Teeth as a Substitute for Human Teeth in Dental Research: A Review of Literature. Journal of Oral Science, 53, 273-282. https://doi.org/10.2334/josnusd.53.273

[24] Titley, K.C., Torneck, C.D., Smith, D.C. and Adibfar, A. (1988) Adhesion of Composite Resin to Bleached and Unbleached Bovine Enamel. Journal of Dental Research, 67, 1523-1528. https://doi.org/10.1177/00220345880670121601

[25] Wennberg, A. and Orstavik, D. (1990) Adhesion of Root Canal Sealers to Bovine Dentine and Gutta-Percha. International Endodontic Journal, 23, 13-19. https://doi.org/10.1111/j.1365-2591.1990.tb00797.x

[26] Schilke, R., Bauß, O., Lisson, J.A., Schuckar, M. and Geurtsen, W. (1999) Bovine Dentin as a Substitute for Human Dentin in Shear Bond Strength Measurements. American Journal of Dentistry, 12, 92-96.

[27] Reis, A.F., Giannini, M., Kavaguchi, A., Soares, C.J. and Line, S.R. (2004) Comparison of Microtensile Bond Strength to Enamel and Dentin of Human, Bovine, and Porcine Teeth. The Journal of Adhesive Dentistry, 6, 117-121.

[28] Zielinski, V., Reimann, S., Jager, A. and Bourauel, C. (2014) Comparison of Shear Bond Strength of Plastic and Ceramic Brackets. Journal of Orofacial Orthopedics, 75, 345-357. https://doi.org/10.1007/s00056-014-0236-6

[29] Anido, A., Amore, R., Lewgoy, H.R. and Anauate-Netto, C. (2012) Comparative Study of Bond Strength to Human and Bovine Dentine at Three Different Depths. British Journal of Dental Science, 15, 56-62.

[30] Evans, L.J., Peters, C., Flickinger, C., Taloumis, L. and Dunn, W. (2002) A Comparison of Shear Bond Strengths of Orthodontic Brackets using Various Light Sources, Light Guides and Cure Times. American Journal of Orthodontics and Dentofacial Orthopedics, 121, 510-515. https://doi.org/10.1067/mod.2002.121558

[31] Oesterle, L.J., Messersmith, M.L., Devine, S.M. and Ness, C.F. (1995) Light and Setting Times of Visible-Light-Cured Orthodontic Adhesives. Journal of Clinical Orthodontics, 29, 31-36.

[32] Brunhano, I.H.V., Fernander, D.J., Sayao de Miranda, M. and Artese, F. (2013) Influence of Surface Treatment on Shear Bond Strength of Orthodontic Brackets. Dental Press Journal of Orthodontics, 18, 54-62. https://doi.org/10.1590/S2176-94512013000300010

[33] [Bishara, S.E., Laffoon, J.F., VonWald, L. and Warren, J.J. (2001) Evaluation of Non-Rinse Conditioning Solution and a Compomer as an Alternative Method of Bonding Orthodontic Bracket. The Angle Orthodontist, 71, 461-465.

[34] Yamamoto, A., Yoshida, T., Tsubota, K., Takamizawa, T., Kurokawa, H. and Miyazaki, M. (2006) Orthodontic Bracket Bonding: Enamel Bond Strength vs. Time. American Journal of Orthodontics and Dentofacial Orthopedics, 130, 435.e1-e6. https://doi.org/10.1016/j.ajodo.2006.03.024

[35] Linn, B.J., Berzins, D.W., Dhuru, V.B. and Bradley, T.G. (2006) A Comparison of Bond Strength between Direct- and Indirect-Bonding Methods. The Angle Orthodontist, 76, 289-294. 